\title{
Time-domain diffuse optical tomography utilizing truncated Fourier series approximation
}

\author{
Meghdoot Mozumder, ${ }^{1,{ }^{*}}$ And Tanja TARvainen, ${ }^{1,2}$ \\ ${ }^{1}$ Department of Applied Physics, University of Eastern Finland P.O. Box 1627, 70211 Kuopio, Finland \\ ${ }^{2}$ Department of Computer Science, University College London, Gower Street, London WC1E 6BT, United \\ Kingdom \\ *meghdoot.mozumder@uef.fi
}

\begin{abstract}
Diffuse optical tomography (DOT) uses near infrared light for in vivo imaging of spatially varying optical parameters in biological tissues. It is known that time-resolved measurements provides the richest information on soft tissues, among other measurement types in DOT such as steady state and intensity modulated measurements. Therefore, several integral transform based moments of the time-resolved DOT measurements has been considered, to estimate spatially distributed optical parameters. However, the use of such moments can result in low contrast images and cross-talks between the reconstructed optical parameters, limiting their accuracy. In this work we propose utilizing a truncated Fourier series approximation in time-resolved DOT. Using this approximation, we obtained optical parameter estimates with accuracy comparable to using whole time-resolved data, using low computational time and resources. The truncated Fourier series approximation based estimates also displayed good contrast and minimal parameter cross-talk, and the estimates further improved in accuracy when multiple Fourier frequencies were used.
\end{abstract}

(C) 2019 Optical Society of America

\section{Introduction}

Near-infrared diffuse optical tomography (DOT) is a non-invasive technique for imaging spatially varying optical properties, typically the absorption and scattering coefficients, in soft biological tissues. The distribution of these optical coefficients are particularly useful in obtaining tissue biochemical and structural information with applications, for example, in early diagnosis of breast cancer [1], in neonatal brain imaging [2-4] and small animal imaging [5].

There are three modalities of DOT based on the type of light source used in the experimental systems: continuous wave DOT, frequency-domain DOT and time-domain DOT. Continuous wave DOT (CW-DOT) systems use continuous wave lasers or lamps for illuminating the tissues. Both absorption and scattering parameters cannot be simultaneously estimated from CW-DOT data, due to non-uniqueness of the image reconstruction problem [6]. Frequency-domain DOT (FD-DOT) uses radio-frequency modulated light, and it has been the most commonly used method for DOT till date. FD-DOT systems employ light sources and detectors which are relatively less expensive than those required for time-domain DOT (TD-DOT) systems [7]. However, recently developed photonic components have demonstrated that compact and wearable TD-DOT, with exceptional depth penetration and sensitivity, can be achieved at relatively low-cost [8]. Also, TD-DOT systems can be used to measure and image larger tissue thickness compared to FD-DOT, and the information content in the measured temporal point spread function is known to be greater than in amplitude and phase measurements performed at one frequency using FD-DOT [7].

TD-DOT was developed during early 1990s', for the first simulation studies see e.g [9], and for the first 32-channel experimental system see [10]. This first system has been applied for imaging human forearm movement [11], imaging newborn infant brains [2-4], for optical mammography [12], and monitoring breast cancer therapy responses [13,14]. Several other TD-DOT systems have been developed for imaging tissue-mimicking phantoms $[15,16]$, for 
imaging brain [17-19] and recently for breast imaging [1].

The image reconstruction problem of TD-DOT involves estimating spatially varying optical parameters, using time-resolved boundary measurements. A few approaches to solve the image reconstruction problem have been proposed. These include, using the whole time-resolved measurement data [20,21], and using moments of the time-resolved measurement data. Here, the moments refer to integral transforms of the measured temporal intensity function, which provides a description or statistics of the temporal function. The various moments considered in TD-DOT are, the first temporal moments (or Mellin transforms) [9,22], Laplace transform [23] and the Mellin-Laplace transform [23,24]. Use of these moments has led to reduction of computation time and memory requirements, due to compression of the time-resolved measurement data [9]. A comparison of estimation accuracies obtained using different choices of moments, using one in silico target, was presented in [24]. It was shown that using only one moment was inadequate to reconstruct both absorption and scattering parameters simultaneously. Specifically, there were inter-parameter cross-talks, such that the absorption and scattering estimates significantly affected each other. It was suggested in Ref. [24] that these effects were due to non-uniqueness of the reconstruction problem similarly as in using intensity data alone [6]. Using a combination of moments was shown to improve results. As such, earlier in vivo studies $[2,3,11,17]$ were carried out using temporal moments such as Mellin transforms, rather than using the whole time-resolved data. The authors in Ref. [3] recognized that the use of moments resulted in parameter cross-talks and thus limited the accuracy of their estimated parameters. A systematic study of the number of moments required for reasonable quality reconstruction, has not been carried out. Ref [24] also demonstrated that the choice of the optimal moment depends on the distribution of target optical properties, which is only partially known in real applications.

Using the whole time-resolved data was shown to be the most accurate method in estimating the optical parameters, compared to using Mellin transforms or combinations of Mellin transforms, in Ref. [20]. However, using the whole time-resolved data required around $1100 \%$ higher computation time, compared to using single moments [20].

Apart from the above mentioned moments, a few other data types have been suggested in the literature, such the integral of time-resolved data over a limited temporal period (time-gating), peak intensity of the data, logarithmic slope of the temporal decay, etc. [24]. These data types only partially use the full temporal information measured, and to our knowledge they have not been studied in detail.

A different approach was proposed by Selb et al. [25], where the TD-DOT sensitivity matrix was calculated by Fourier transforming the FD-DOT sensitivity matrices obtained at 101 frequencies. This approach was computationally relatively intensive, since 101 sensitivity matrices corresponding to each Fourier frequency, were required in the estimation. A relatively faster approach was proposed by Gibson et al. [4], where the measurement data was Fourier transformed to one particular frequency, to reconstruct optical parameter changes due to brain activation. The method presented in [4] used difference imaging, by subtracting the brain activation data with the resting stage data. As we later demonstrate in this work, the method used in [4] does not account for the finite pulse width of the source, and as such cannot be applied for absolute imaging of the optical parameters.

In this article we propose an approach for reconstruction of optical parameters in TD-DOT utilising truncated Fourier-series approximation. The approach is based on exact modelling of Fourier transformed measurements. It includes modelling of the temporal length of the source pulses and noise in the Fourier transformed measurements, unlike the previously proposed methods using Fourier transform [4] or other moments [24]. Furthermore, the methodology enables choice of frequencies that are used in the solution of the image reconstruction problem.

The paper is organized as follows. We describe the measurement model, reconstruction methods and simulations in Section 2. The results of the simulations are presented on Section 3. 
The conclusions are presented in Section 4.

\section{Theory}

\subsection{Time-domain diffuse optical tomography}

In a typical TD-DOT measurement setup near-infrared light is introduced into an object from the boundary of the object. Let $\Omega \subset \mathbb{R}^{d}$, where $d$ is the dimension of the medium $(=2,3)$, model this object domain. In a diffuse medium like biological tissue, the commonly used light transport model for TD-DOT is the diffusion approximation (DA) to the radiative transport equation $[26,27]$

$$
\begin{gathered}
\left(-\nabla \cdot \frac{1}{d\left(\mu_{\mathrm{a}}(r)+\mu_{\mathrm{s}}^{\prime}(r)\right)} \nabla+\mu_{\mathrm{a}}(r)+\frac{1}{c} \frac{\partial}{\partial t}\right) \Phi(r, t)=0 \quad r \in \Omega \\
\Phi(r, t)+\frac{1}{2 \gamma d\left(\mu_{\mathrm{a}}(r)+\mu_{\mathrm{s}}^{\prime}(r)\right)} \alpha \frac{\partial \Phi(r, t)}{\partial \hat{k}}= \begin{cases}\frac{Q}{\gamma}(r, t) & r \in s \\
0 & r \in \partial \Omega \backslash s\end{cases}
\end{gathered}
$$

where $\Phi(r, t)$ is the photon fluence, $\mu_{\mathrm{a}}(r)$ is the absorption coefficient and $\mu_{\mathrm{s}}^{\prime}(r)$ is the (reduced) scattering coefficient. Further, $c$ is the speed of light in the medium, parameter $Q(r, t)$ is the strength of the pulsed (temporal) light source at boundary locations $s \subset \partial \Omega$. The parameter $\gamma$ is a dimension dependent constant $\left(\gamma=1 / \pi\right.$ when $\Omega \subset \mathbb{R}^{2}, \gamma=1 / 4$ when $\left.\Omega \subset \mathbb{R}^{3}\right)$ and $\alpha$ is a parameter governing the internal reflection at the boundary $\partial \Omega$. The measurable quantity exitance $\Gamma(r, t)$ is given by

$$
\Gamma(r, t)=-\frac{1}{d\left(\mu_{\mathrm{a}}(r)+\mu_{\mathrm{s}}^{\prime}(r)\right)} \frac{\partial \Phi(r, t)}{\partial \hat{k}}=\frac{2 \gamma}{\alpha} \Phi(r, t),
$$

where $\hat{k}$ is the outward normal to the boundary.

In this work, the numerical approximation of the model (1)-(3) is based on a finite element (FE) approximation, following the framework derived in [28,29]. In the FE-approximation, the domain $\Omega$ is divided into $\mathrm{N}_{\mathrm{e}}$ non-overlapping elements joined at $\mathrm{N}_{\mathrm{n}}$ vertex nodes. The photon fluence at time instant $t$, in a finite dimensional basis is given by

$$
\Phi^{\mathrm{h}}(r, t)=\sum_{k=1}^{\mathrm{N}_{\mathrm{n}}} \phi_{k}(t) \psi_{k}(r) \in \Omega^{\mathrm{h}},
$$

where $\Omega^{\mathrm{h}}$ is a finite dimensional subspace spanned by basis functions $\psi_{k}, k=1 \ldots \mathrm{N}_{\mathrm{n}}$, and $\phi_{k}(t), k=1 \ldots \mathrm{N}_{\mathrm{n}}$ are the photon density at the $\mathrm{N}_{\mathrm{n}}$ nodes of the FE mesh. We write finite dimensional approximations for $\mu_{\mathrm{a}}(r)$ and $\mu_{\mathrm{s}}^{\prime}(r)$

$$
\mu_{\mathrm{a}}(r)=\sum_{l=1}^{\mathrm{N}_{\mathrm{n}}} \mu_{\mathrm{a}, l} \psi_{l}(r), \mu_{\mathrm{s}}^{\prime}(r)=\sum_{l=1}^{\mathrm{N}_{\mathrm{n}}} \mu_{\mathrm{s}, l} \psi_{l}(r),
$$

where $\mu_{\mathrm{a}, l}, \mu_{\mathrm{s}, l}$ denote the absorption and scattering at $\mathrm{N}_{\mathrm{n}}$ nodes of the FE mesh.

The FE-approximation of the time-domain diffusion equation (1)-(2) is

$$
\mathrm{K} \Phi^{\mathrm{h}}(t)+\mathrm{M} \frac{\partial \Phi^{\mathrm{h}}(t)}{\partial t}=\mathrm{Q}(t) .
$$


where matrices $\mathrm{K}$ and $\mathrm{M}$ and vector $\mathrm{Q}(t)$ are

$$
\begin{aligned}
\mathrm{K}_{m k}= & \int_{\Omega} \sum_{l=1}^{\mathrm{N}_{\mathrm{n}}}\left[\frac{1}{d\left(\mu_{\mathrm{a}, l}(r)+\mu_{\mathrm{s}, l}(r)\right)} \psi_{l}(r) \nabla \psi_{m}(r) \cdot \nabla \psi_{k}(r)\right] \mathrm{d} r \\
& +\int_{\Omega} \sum_{l=1}^{\mathrm{N}_{\mathrm{n}}} \mu_{\mathrm{a}, l}(r) \psi_{l}(r) \psi_{m}(r) \psi_{k}(r) \mathrm{d} r+\frac{2 \gamma}{\alpha} \int_{\partial \Omega} \psi_{m}(r) \psi_{k}(r) \mathrm{d} r \\
\mathrm{M}_{m k}= & \int_{\Omega} \psi_{m}(r) \psi_{k}(r) \mathrm{d} r, \\
\mathrm{Q}_{m}(t)= & \int_{\partial \Omega} Q(r, t) \psi_{m}(r) \mathrm{d} r,
\end{aligned}
$$

where $m$ and $k$ are the nodal indices. In this work, we use a finite-difference method to integrate Eq. (6) for a sequence of time steps, $t_{i}=i \Delta t(i=1,2, \ldots)$

$$
\left[\theta \mathrm{K}+\frac{1}{\Delta t} \mathrm{M}\right] \Phi^{h}\left(t_{i+1}\right)+\left[(1-\theta) \mathrm{K}-\frac{1}{\Delta t} \mathrm{M}\right] \Phi^{h}\left(t_{i}\right)=\theta \mathrm{Q}\left(t_{i+1}\right)+(1-\theta) \mathrm{Q}\left(t_{i}\right),
$$

where parameter $\theta$ controls the coupling of adjacent time steps. Here, we set $\theta=0.5$, which corresponds to the Crank-Nicholson scheme. A typical TD-DOT measurement setup collects the time-varying boundary exitance $\Gamma(t), t=1, \ldots, \mathrm{T}$, Eq. (3), where $\mathrm{T}$ is the temporal range of the output signal.

In practice, the measured data is corrupted by noise. In the case of additive noise, a discretized observation model for TD-DOT is

$$
y=A_{t}\left(\mu_{\mathrm{a}}, \mu_{\mathrm{s}}^{\prime}\right)+e
$$

where $A_{t}$ denotes the FE solution of $\Gamma(t), \mathrm{y} \in \mathbb{R}^{\mathrm{N}_{\mathrm{m}}}$ is data vector, $\mathrm{N}_{\mathrm{m}}$ is the number of measurements, $e \in \mathbb{R}^{N_{\mathrm{m}}}$ denotes the random noise in measurements, and $\left(\mu_{\mathrm{a}}, \mu_{\mathrm{s}}^{\prime}\right) \in \mathbb{R}^{2 \mathrm{~N}_{\mathrm{n}}}$ are the discretized optical coefficients, Eq. (5).

\subsection{Truncated Fourier series approximation}

Let us consider a delta function (in time) source at the boundary, and denote the corresponding boundary excitance as $\Gamma_{\delta}(t)$. The truncated Fourier series approximation of the time-resolved boundary excitance $\Gamma_{\delta}(t)$ is given by [30],

$$
\Gamma_{\delta}(t)=\sum_{k=-\mathrm{N}_{\omega}}^{\mathrm{N}_{\omega}} \Gamma\left(\omega_{k}\right) \exp \left(\mathrm{i} \omega_{k} t\right),
$$

where $\mathrm{i}$ is the imaginary unit, $\mathrm{N}_{\omega}$ is the number of Fourier frequencies $\omega_{k}=2 \pi k /$ T involved in the approximation, $\Gamma\left(\omega_{k}\right)$ are the Fourier coefficients computed with operator $\mathcal{L}(\cdot)$ as

$$
\Gamma\left(\omega_{k}\right)=\mathcal{L}\left(\Gamma_{\delta}(t)\right)=\frac{1}{\mathrm{~T}} \int_{0}^{\mathrm{T}} \Gamma_{\delta}(t) \exp \left(-\mathrm{i} \omega_{k} t\right) \mathrm{d} t
$$

The temporal range of the point-spread function $\Gamma(t)$ is $[0, \mathrm{~T}]$. For light sources with a finite temporal length, the measurable output $\Gamma(t)$ can be expressed as a convolution $(*)$ of the source $Q(t)$ and exitance due to delta source $\Gamma_{\delta}(t)$ as,

$$
\Gamma(t)=\Gamma_{\delta}(t) * Q(t)
$$


implying that taking a Fourier transform results in the pointwise product $(\cdot)$ of their Fourier transforms (by convolution theorem) as,

$$
\mathcal{L}(\Gamma(t))=\mathcal{L}\left(\Gamma_{\delta}(t)\right) \cdot \mathcal{L}(Q(t))
$$

As such, we can formulate an observation model for a Fourier-transform $y_{\mathrm{FT}}$ of a measured noisy data $y$

$$
y_{\mathrm{FT}}=\left(\begin{array}{l}
\operatorname{Re} \mathcal{L}(y) \\
\operatorname{Im} \mathcal{L}(y)
\end{array}\right)=\left(\begin{array}{l}
\operatorname{Re} \mathcal{L}(\Gamma(t)+e) \\
\operatorname{Im} \mathcal{L}(\Gamma(t)+e)
\end{array}\right)=\left(\begin{array}{l}
\operatorname{Re}\left(\Gamma\left(\omega_{k}\right)\right) \\
\operatorname{Im}\left(\Gamma\left(\omega_{k}\right)\right)
\end{array}\right) \cdot \underbrace{\left(\begin{array}{l}
\operatorname{Re}(\mathcal{L}(Q(t))) \\
\operatorname{Im}(\mathcal{L}(Q(t)))
\end{array}\right)}_{Q_{\mathrm{FT}}}+\underbrace{\left(\begin{array}{l}
\operatorname{Re}(\mathcal{L}(e)) \\
\operatorname{Im}(\mathcal{L}(e))
\end{array}\right)}_{e_{\mathrm{FT}}},
$$

where $Q_{\mathrm{FT}}$ is the Fourier transform of the source pulse and $e_{\mathrm{FT}}$ is the Fourier transform of the noise. We can then construct the normalized measurement data

$$
\begin{aligned}
\tilde{y}=\frac{y_{\mathrm{FT}}}{Q_{\mathrm{FT}}} & =\left(\begin{array}{l}
\operatorname{Re}\left(\Gamma\left(\omega_{k}\right)\right) \\
\operatorname{Im}\left(\Gamma\left(\omega_{k}\right)\right)
\end{array}\right)+\frac{e_{\mathrm{FT}}}{Q_{\mathrm{FT}}}, \\
& =A_{\omega}\left(\mu_{\mathrm{a}}, \mu_{\mathrm{s}}^{\prime}\right)+\tilde{e}
\end{aligned}
$$

where the normalized data $\tilde{y}$, now corresponds to noisy measurements carried out at frequencies $\omega_{k}, \tilde{e}=e_{\mathrm{FT}} / Q_{\mathrm{FT}}$ is the random noise, and $A_{\omega}$ can be numerically modelled using the frequencydomain diffusion approximation model $[9,31,32]$ as

$$
\mathrm{K} \Phi^{\mathrm{h}}\left(\omega_{k}\right)+\frac{\mathrm{i} \omega_{k}}{c} \mathrm{M} \Phi^{\mathrm{h}}\left(\omega_{k}\right)=\mathrm{Q}\left(\omega_{k}\right)
$$

The matrices K, M are defined in Eqs. (7),(8), and the source vector $\mathrm{Q}\left(\omega_{k}\right)$ is given by

$$
\mathrm{Q}_{m n}\left(\omega_{k}\right)=\int_{\Omega} Q\left(r, \omega_{k}\right) \psi_{m}(r) \psi_{n}(r) \mathrm{d} r,
$$

where $Q\left(r, \omega_{k}\right)$ is an amplitude-modulated source with an angular modulation frequency $\omega_{k}$.

In comparison to using the whole time-domain measurement data, which requires matrix multiplications and divisions at each time step (Eq. (10)), the measurement model (17) requires only one of these operations at each frequency, thereby reducing the computational requirements significantly, when only a few Fourier frequencies are used. Also, in contrast to earlier proposed temporal moments [9,24], the truncated Fourier series approximation model (17) explicitly models the finite temporal source length and the measurement noise in the transformed data.

\subsection{Bayesian approach to the inverse problem of TD-DOT}

In the Bayesian approach to inverse problems, all the parameters are considered random variables and the uncertainty of their values is encoded into probability density models [31-33]. Let us consider the TD-DOT observation model Eq. (11). The solution of the inverse problem is the posterior probability density which is obtained through Bayes' theorem and can be written as

$$
\pi\left(\mu_{\mathrm{a}}, \mu_{\mathrm{s}}^{\prime} \mid y\right) \propto \pi\left(y \mid \mu_{\mathrm{a}}, \mu_{\mathrm{s}}^{\prime}\right) \pi\left(\mu_{\mathrm{a}}, \mu_{\mathrm{s}}^{\prime}\right)
$$

where $\pi\left(y \mid \mu_{\mathrm{a}}, \mu_{\mathrm{s}}^{\prime}\right)$ is the likelihood density and $\pi\left(\mu_{\mathrm{a}}, \mu_{\mathrm{s}}^{\prime}\right)$ is the prior density. The posterior density $\pi\left(\mu_{\mathrm{a}}, \mu_{\mathrm{s}}^{\prime} \mid y\right)$ is a probability density in a very high-dimensional space. Since we aim at computationally efficient solutions, we compute point estimate(s) from the posterior density, 
the most typical choice being the maximum a posteriori (MAP) estimate. Assuming that the unknowns $\mu_{\mathrm{a}}$ and $\mu_{\mathrm{s}}^{\prime}$ and noise $e$ are mutually independent and Gaussian distributed, i.e

$$
\mu_{\mathrm{a}} \sim \mathcal{N}\left(\mu_{a, *}, \Gamma_{\mu_{\mathrm{a}}}\right), \quad \quad \mu_{\mathrm{s}}^{\prime} \sim \mathcal{N}\left(\mu_{s, *}^{\prime}, \Gamma_{\mu_{\mathrm{s}}^{\prime}}\right), \quad e \sim \mathcal{N}\left(e_{*}, \Gamma_{e}\right),
$$

where $\mu_{a, *}, \mu_{s, *}$ and $e_{*}$ are the means, and $\Gamma_{\mu_{\mathrm{a}}}, \Gamma_{\mu_{\mathrm{s}}^{\prime}}$ and $\Gamma_{e}$ are the covariance matrices, the MAP estimate is obtained as

$$
\left(\hat{\mu}_{\mathrm{a}}, \hat{\mu}_{\mathrm{s}}^{\prime}\right)=\underset{\mu_{\mathrm{a}}, \mu_{\mathrm{s}}^{\prime}}{\arg \min }\left\{\left\|L_{e}\left(y-A_{t}\left(\mu_{\mathrm{a}}, \mu_{\mathrm{s}}^{\prime}\right)-e_{*}\right)\right\|^{2}+\left\|L_{\mu_{\mathrm{a}}}\left(\mu_{\mathrm{a}}-\mu_{a, *}\right)\right\|^{2}+\left\|L_{\mu_{\mathrm{s}}^{\prime}}\left(\mu_{\mathrm{s}}^{\prime}-\mu_{s, *}^{\prime}\right)\right\|^{2}\right\},
$$

where the Cholesky factors are $L_{\mu_{\mathrm{a}}}^{\mathrm{T}} L_{\mu_{\mathrm{a}}}=\Gamma_{\mu_{\mathrm{a}}}^{-1}, L_{\mu_{\mathrm{s}}^{\prime}}^{\mathrm{T}} L_{\mu_{\mathrm{s}}^{\prime}}=\Gamma_{\mu_{\mathrm{s}}^{\prime}}^{-1}$ and $L_{e}^{\mathrm{T}} L_{e}=\Gamma_{e}^{-1}$.

When considering the truncated Fourier series approximation based observation model, Eq. (17), with Gaussian distributed $\mu_{\mathrm{a}}$ and $\mu_{\mathrm{s}}^{\prime}$, and Gaussian distributed noise

$$
\tilde{e} \sim \mathcal{N}\left(\tilde{e}_{*}, \Gamma_{\tilde{e}}\right)
$$

the MAP estimate is obtained as

$$
\left(\hat{\mu}_{\mathrm{a}}, \hat{\mu}_{\mathrm{s}}^{\prime}\right)=\underset{\mu_{\mathrm{a}}, \mu_{\mathrm{s}}^{\prime}}{\arg \min }\left\{\left\|L_{\tilde{e}}\left(\tilde{y}-A_{\omega}\left(\mu_{\mathrm{a}}, \mu_{\mathrm{s}}^{\prime}\right)-\tilde{e}_{*}\right)\right\|^{2}+\left\|L_{\mu_{\mathrm{a}}}\left(\mu_{\mathrm{a}}-\mu_{a, *}\right)\right\|^{2}+\left\|L_{\mu_{\mathrm{s}}^{\prime}}\left(\mu_{\mathrm{s}}^{\prime}-\mu_{s, *}^{\prime}\right)\right\|^{2}\right\},
$$

where the Cholesky factor $L_{\tilde{e}}^{\mathrm{T}} L_{\tilde{e}}=\Gamma_{\tilde{e}}^{-1}$.

In this work, the MAP estimates (21) and (22) were calculated using the Gauss-Newton method with a positivity constraint and a line search for determining the step length. The calculation of the Jacobians for the Gauss-Newton algorithm are described in Appendix A. The relative errors,

$$
\mathrm{E}_{x}=\frac{\left\|x_{\mathrm{MAP}}-x_{\text {Target }}\right\|}{\left\|x_{\text {Target }}\right\|} \cdot 100 \%,
$$

where $x_{\text {MAP }}$ are the MAP estimates and $x_{\text {Target }}$ are the target optical parameters, was used to evaluate the accuracy of the estimation.

\subsubsection{Prior model}

In this work we use Gaussian Ornstein-Uhlenbeck prior [34]. The covariance matrix of the Ornstein-Uhlenbeck prior between nodes $m$ and $k$ of a FE-mesh is defined as

$$
\Gamma_{x, m k}=\sigma_{x}^{2} \exp \left(-\frac{\left\|r_{m}-r_{k}\right\|}{l}\right)
$$

where $x$ denotes the optical coefficients, $\sigma_{x}$ the corresponding variances, $r_{m}, r_{k}$ are the nodal locations and $l$ is the characteristic length scale which controls the spatial range of correlation. For calculating the MAP estimates, the means $\left(\mu_{a, *}, \mu_{s, *}\right)$ were set as the background optical parameters, the standard deviations $\left(\sigma_{\mu_{\mathrm{a}}}, \sigma_{\mu_{\mathrm{s}}^{\prime}}\right)$ were set such that maximum target values corresponded to two standard deviations from the background. The characteristic length scale was set as $16 \mathrm{~mm}$, corresponding approximately to the size of the inhomogenities in the targets used.

\section{Simulations}

The computations were carried out in a Fujitsu Celcius W550 desktop workstation, with Intel@Xeon(R) W-2125 CPU @ 4.00GHz×8, using MATLAB (R2017b, Mathworks, Natick, MA). The Toast++ software [35] was utilized in the FE-solution of the diffusion equation. 


\subsection{Data generation}

In the numerical studies, the domain $\Omega \subset \mathbb{R}^{2}$ was a circle with a radius of $25 \mathrm{~mm}$. The measurement setup consisted of 16 sources and 16 detectors. The source and detector optodes were modeled as Gaussian surface patches with $2 \mathrm{~mm}$ width, located at equi-spaced angular intervals on the boundary $\partial \Omega$.

First, we studied a target with background optical properties $\mu_{\mathrm{a}}=0.01 \mathrm{~mm}^{-1}, \mu_{\mathrm{s}}^{\prime}=1 \mathrm{~mm}^{-1}$, containing two absorption inclusions with $\mu_{\mathrm{a}}=0.02 \mathrm{~mm}^{-1}, \mu_{\mathrm{a}}=0.005 \mathrm{~mm}^{-1}$ and two scatter inclusions with $\mu_{\mathrm{s}}^{\prime}=2 \mathrm{~mm}^{-1}, \mu_{\mathrm{s}}^{\prime}=0.5 \mathrm{~mm}^{-1}$, as shown in the first column of Fig. 1 .

For the second test, we studied randomly simulated targets. Therefore, 50 separate draws of optical parameter distributions, from the prior model (24) were used to simulate optical parameters and to generate data. An example of one sample drawn is shown in the first column of Fig. 2. These were used to test the performance of the proposed truncated Fourier series approximation model (17), using multiple Fourier frequencies.

For all simulated targets, the time-resolved data was simulated using FE-approximation of the DA in a mesh with 33806 nodes and 67098 triangular elements. The time discretization was 0.1 pico-second (ps), the source pulses had $10 \mathrm{ps}$ duration, and the temporal range was specified as 5000 ps. The total number of measurements $\mathrm{N}_{\mathrm{m}}$ were 12800000 ( 256 combination of sources and detectors, 50000 time steps). Random measurement noise $e$, that was drawn from a zero-mean Gaussian distribution

$$
\pi(e)=\mathcal{N}\left(0, \Gamma_{e}\right), \quad \Gamma_{e}=\operatorname{diag}\left(\sigma_{e, 1}^{2}, \ldots, \sigma_{e, \mathrm{~N}_{\mathrm{m}}}^{2}\right)
$$

where the standard deviations $\sigma_{e, 1}^{2}, \ldots, \sigma_{e, \mathrm{~N}_{\mathrm{m}}}$ were specified as $1 \%$ of the simulated noise free measurement data, was added to the simulated measurement data. In real experiments, more complicated noise can be expected due to the laser fluctuations, thermal noise, etc. However, if many measurement noise samples can be obtained, a Gaussian approximation for the noise model could be sufficient, due to the central limit theorem. Errors due to laser source instabilities and detector sensitivities can also be calibrated or marginalized in DOT separately, see e.g. [31] and the references therein.

\subsection{Estimation}

\subsubsection{Using multiple Fourier frequencies}

For calculating the MAP estimates with the truncated Fourier series approximation (17), we used a FE-mesh with 26075 nodes and 51636 elements, and sampling the measurements at 1ps. The modelling errors due to discretisation and sampling in this case were lower than the additive measurement error (25), implying that the MAP estimation (22) effectively marginalized the modelling errors. We studied the estimation accuracies utilizing different number of Fourier frequencies.

For calculating the MAP estimates (22), the noise means and covariances were assumed known. Note that the distribution of $\tilde{e}$, given the distribution of $e(25)$ is

$$
\pi(\tilde{e})=\mathcal{N}\left(0, \Gamma_{\tilde{e}}\right), \quad \Gamma_{\tilde{e}}=\operatorname{diag}\left(\left[\operatorname{Re}\left(\mathcal{L}\left(\sigma_{e, 1}^{2}, \ldots, \sigma_{e, 2 \mathrm{~N}_{\mathrm{m}}}^{2}\right)\right)\right],\left[\operatorname{Im}\left(\mathcal{L}\left(\sigma_{e, 1}^{2}, \ldots, \sigma_{e, 2 \mathrm{~N}_{\mathrm{m}}}^{2}\right)\right)\right]\right) .
$$

\subsubsection{Comparison to reference estimates}

We compared the estimates obtained using the truncated Fourier series approximation model to reference estimates using the whole time-resolved measurement data $y$, Eq. (11). Due to computational limitations, a mesh with a lower discretization was used, with 379 nodes and 702 elements. To ensure that these reference estimates were devoid of modelling errors, the same FE mesh was used to generate the data and calculate MAP estimates (21), and the measurements were sampled at the same time interval they were generated (at $0.1 \mathrm{ps}$ intervals). 


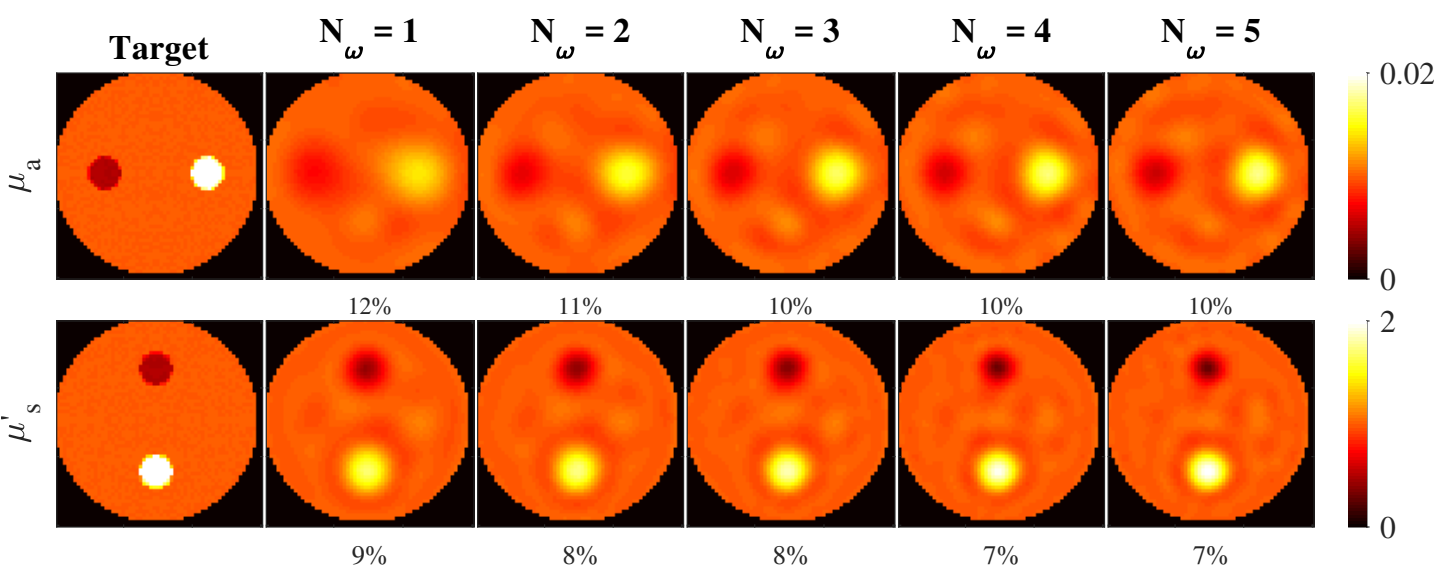

Fig. 1. Absorption (first row) and scattering (second row) distributions. First column: simulated (true) target. Columns 2-6 from left to right: estimated parameters using one to five Fourier frequencies $\left(\mathrm{N}_{\omega}=1, \ldots, 5\right)$. The numbers below the images indicate the relative errors of the estimates, Eq. (23).

\section{Results}

\subsection{Using multiple Fourier frequencies}

MAP estimates obtained using multiple Fourier frequencies $\left(\mathrm{N}_{\omega}=1, \ldots, 5\right)$ and using the first target object is displayed in Fig. 1. The MAP estimate using one example target drawn from the prior model (24) is shown in Fig. 2. The relative errors (23) of these estimates are displayed below each image.

We observe that the estimates obtained using the proposed truncated Fourier transform model (17) show minimal parameter cross-talk, and provide estimates with similar contrast to target objects, unlike previously proposed temporal moments or data types [20,24]. The previously proposed temporal moments were inadequate to reconstruct both absorption and scattering parameters, possibly due to non-uniqueness in the image reconstruction problem using only intensity data [24]. In contrast, the proposed method used both intensity and phase of the Fourier transformed measurements, which very likely alleviates the non-uniqueness issues. Furthermore, it can be noticed that the relative errors of the estimates slightly decrease as the number of the Fourier frequencies increase. However, after three or four frequencies both image quality and relative errors do not clearly change even if the number of Fourier components is increased.

The computational time of the estimates in Fig. 1 are given in Table 1. We observe an increase in computation time with increasing number of Fourier frequencies. This is because, as the number of Fourier frequencies increases, the forward model (18) and Jacobian, needs to be calculated separately for each Fourier frequency.

The statistics of the estimation errors using 50 targets drawn from the prior (24), using multiple Fourier frequencies, are shown in Fig. 3. The parameter maps in Figs. 1, 2, and estimation accuracies in Fig. 3, appear improved on using multiple Fourier frequencies. However, from the statistics in Fig. 3, we observe that the medians and standard deviations of the estimation errors don't change considerably after four Fourier frequencies, especially for the more interesting absorption parameter $\left(\mu_{\mathrm{a}}\right)$.

\subsection{Comparison to reference estimates and discussion}

The reference MAP estimates obtained using full time-domain data are shown in Fig. 4. The figure also shows the estimates obtained four Fourier frequencies $\left(\mathrm{N}_{\omega}=4\right)$. The relative errors 


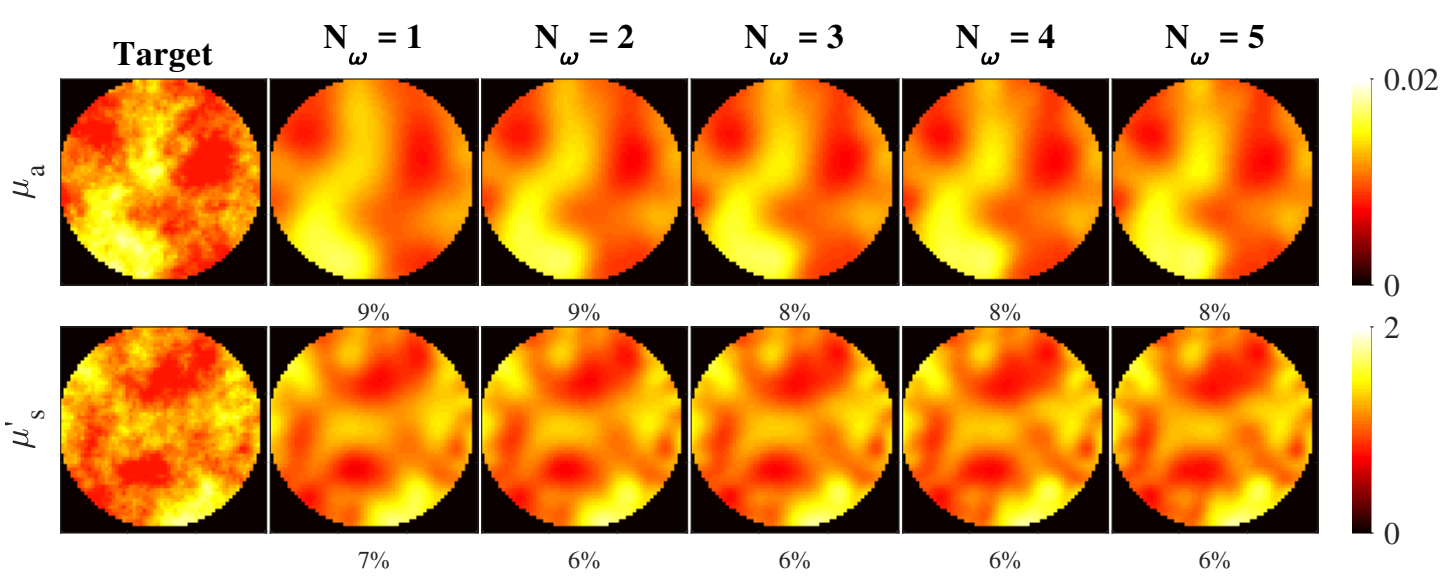

Fig. 2. Absorption (first row) and scattering (second row) distributions. First column: simulated (true) target. Columns 2-6 from left to right: estimated parameters using one to five Fourier frequencies $\left(\mathrm{N}_{\omega}=1, \ldots, 5\right)$. The numbers below the images indicate the relative errors of the estimates, Eq. (23).
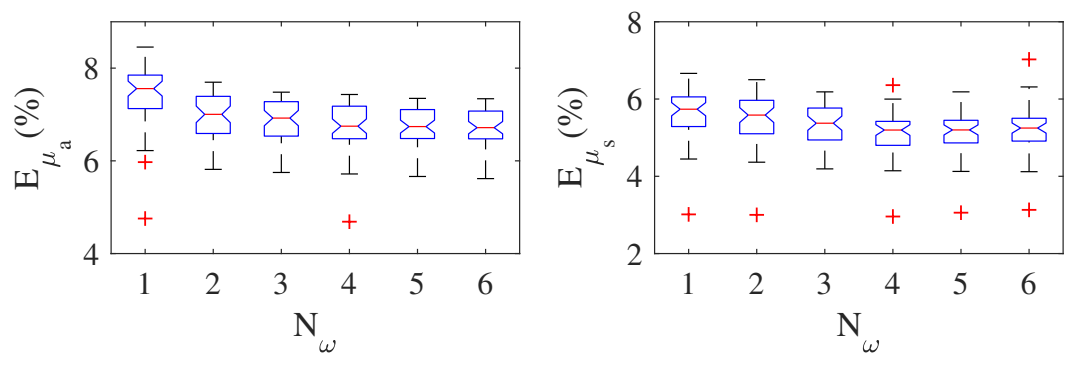

Fig. 3. Statistics of estimation errors using 50 targets drawn from the prior, and using multiple Fourier frequencies. The boxplots represent the distribution of the relative estimation error in estimated absorption coefficient $\mathrm{E}_{\mu_{\mathrm{a}}}$ (left image) and, scattering coefficient $\mathrm{E}_{\mu_{\mathrm{s}}^{\prime}}$ (right image), using one to six Fourier frequencies $\left(\mathrm{N}_{\omega}=1, \ldots, 6\right)$. On each boxplot, the central red mark indicates the median, and the bottom and top blue edges of the box indicate the 25 th and 75 th percentiles, respectively. The outliers are plotted individually using the red '+' symbol.

Table 1. Computational times $\mathrm{t}(\mathrm{s})$, of reference estimates, and estimates with the truncated Fourier series approximation using $\mathrm{N}_{\omega}=1, \ldots, 5$.

\begin{tabular}{lllllll}
\hline & Reference & $\mathrm{N}_{\omega}=1$ & $\mathrm{~N}_{\omega}=2$ & $\mathrm{~N}_{\omega}=3$ & $\mathrm{~N}_{\omega}=4$ & $\mathrm{~N}_{\omega}=5$ \\
\hline $\mathrm{t}(\mathrm{s})$ & 18624 & 21 & 18 & 45 & 70 & 92
\end{tabular}


(23) are given below each image. As it can be seen, the truncated Fourier transform estimate matches closely to the target optical properties and also to the reference estimate.

The computation time for calculating the reference estimate is given in Table 1. We can see that the proposed approach utilizing truncated Fourier series approximation (17) used considerable lower computation time, compared to using the whole measurement data. Even though, for estimating using the whole measurement data, we used a mesh with much lower discretisation. As such, the truncated Fourier transform model (17) provides an effective data compression and reconstruction technique for TD-DOT, allowing estimation of optical parameters, with good accuracy, using low computational resources.

We observe from the statistics of estimation errors in Fig. 3 and computation times in Table 1, that the estimation errors do not change considerably after four Fourier frequencies, in comparison to the loss in computational efficiency. Also, the reconstruction accuracy using four Fourier frequencies is almost comparable to the reference estimate, presented in Fig. 4. However, it should be noted that although four frequencies could be optimal in this case, the target optical properties can be expected to affect on the number of frequencies required.

The temporal point spread function (TPSF) of a data simulated with a target with constant optical properties and the corresponding power spectrum are shown in Fig. 5. In addition, TPSFs of a truncated Fourier series approximation of the data approximated using different number of frequencies are shown. The data was simulated in a two-dimensional circle with a radius of $25 \mathrm{~mm}$, with constant optical parameters $\mu_{\mathrm{a}}=0.01 \mathrm{~mm}^{-1}, \mu_{\mathrm{s}}^{\prime}=1 \mathrm{~mm}^{-1}$. The source and measurement locations were on the opposite sides of the domain. We can observe in Fig. 5 that a few Fourier frequencies are enough to reconstruct the whole point spread function. This is also reflected in the power spectra shown on the right image, where the spectral energy distribution is mainly in the first few frequencies. However, it can also be noticed that, although four frequencies could be used to produce as good quality reconstructions as with the whole time-domain data, it is not enough to capture the TPSF exactly.

Reconstructions in a 3D geometry are shown in Appendix B. The computation times using the 3D mesh was slightly higher, due to the larger number of mesh nodes and elements. However, the number of Fourier frequencies required for reasonable reconstructions in this case was also low.

Compared to previously proposed Fourier transform based method in Ref. [4], the proposed method works independent of source pulse width and models the measurement noise accurately. For a more detailed comparison, see Appendix C.

Future work will include comparison of the truncated Fourier series approximation method to previously proposed moments and data types. We will also consider parallelization of the computations of the forward model and Jacobians of the independent Fourier frequencies. Furthermore, these methods will be evaluated with experimental TD-DOT data.

\section{Conclusions}

We have shown the feasibility of the truncated Fourier series approximation in reliable estimation of spatially varying optical coefficients using TD-DOT. The approach was tested with 2D and 3D simulations, and comparing against a reference estimate using a whole time-domain data. Further, the estimation accuracy using several Fourier frequencies was also analyzed. The results show that the proposed approach can recover reliable estimates of optical parameters, using only few Fourier frequencies ( $\sim 4)$, using significantly low computational resources. Based on these results, we suggest that the truncated Fourier series approximation could provide an efficient modeling protocol in practical TD-DOT imaging situations.

\section{Disclosures}

The authors declare that there are no conflicts of interest related to this article. 


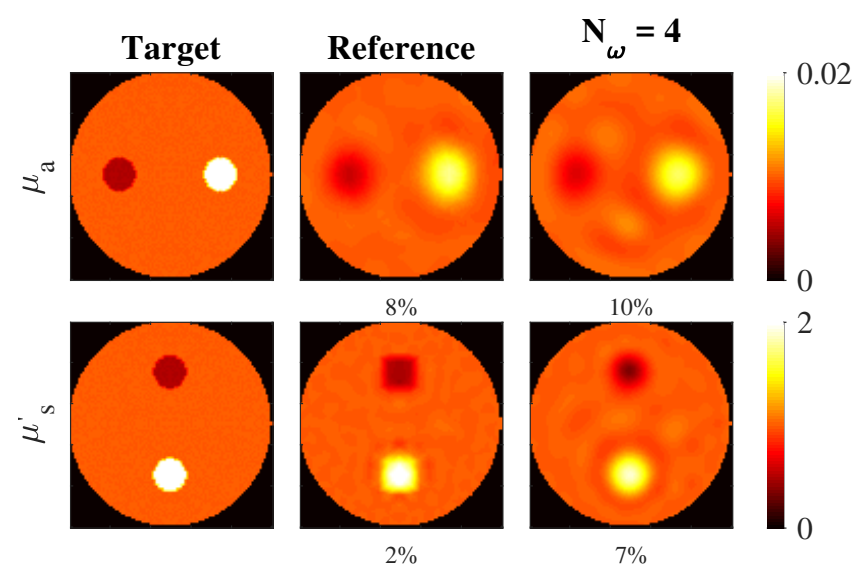

Fig. 4. Absorption (first row) and scattering (second row) distributions. First column: simulated (true) target. Second column: reference estimates computed using whole timedomain data. Third column: estimates computed using four Fourier frequencies. The numbers below the images indicate the relative errors of the estimates, Eq. (23).
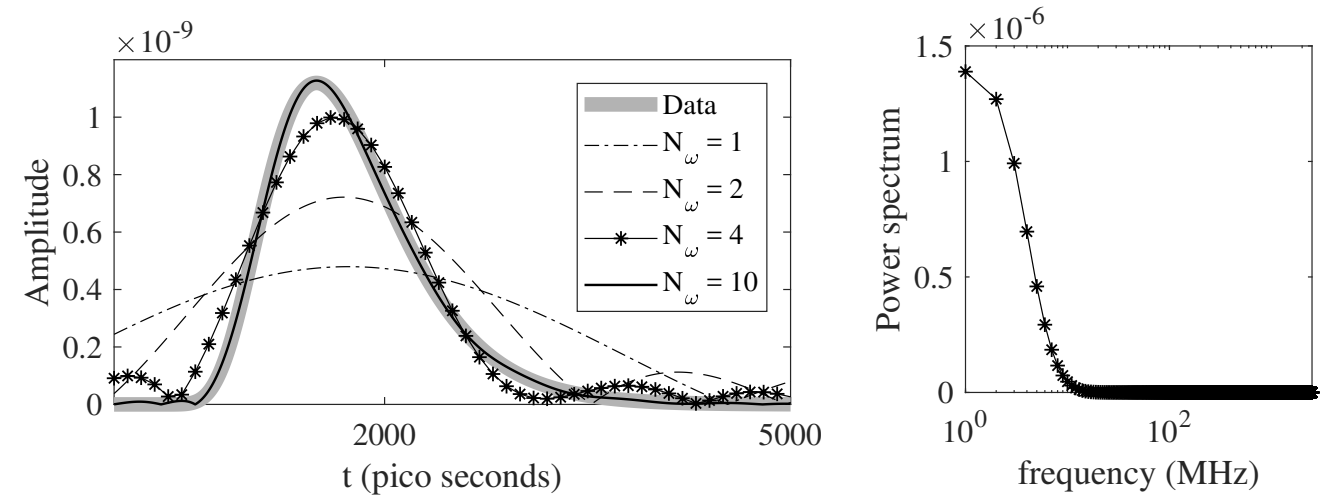

Fig. 5. Left image: Temporal-point spread function (TPSF) of data (bold grey line) and the reconstructed TPSFs using 1, 2, 4 and 10 Fourier frequencies (black lines). Right image: The power spectrum of the data. 


\section{Appendix A: Calculation of Jacobian}

We present here the FE-based Jacobians for the whole time-domain observation model (11), and for the truncated Fourier series approximation model (17). For this, we explicitly differentiate the time-domain FE-approximation (6) with respect to $\mu_{\mathrm{a}}$ and $\mu_{\mathrm{s}}^{\prime}$. We obtain the following recursive relations by differentiating Eq. (6),

$$
\frac{\partial \Phi^{h}\left(t_{i+1}\right)}{\partial \mu}=-\left[\theta \mathrm{K}+\frac{1}{\Delta t} \mathrm{M}\right]^{-1}\left[\theta \frac{\partial \mathrm{K}}{\partial \mu} \Phi^{h}\left(t_{i+1}\right)+\left[(1-\theta) \mathrm{K}-\frac{1}{\Delta t} \mathrm{M}\right] \frac{\partial \Phi^{h}\left(t_{i}\right)}{\partial \mu}+(1-\theta) \frac{\partial K}{\partial \mu} \Phi^{h}\left(t_{i}\right)\right] .
$$

where $\mu$ is either $\mu_{\mathrm{a}}$ or $\mu_{\mathrm{s}}^{\prime}$, matrices $\mathrm{K}$ and $\mathrm{M}$ are defined as in Eqs. (7),(8), and $\frac{\partial \mathrm{K}}{\partial \mu}$ for $\mu=\mu_{\mathrm{a}}$ and $\mu=\mu_{\mathrm{s}}^{\prime}$ are,

$$
\begin{aligned}
\frac{\partial \mathrm{K}}{\partial \mu_{\mathrm{a}}} & =\int_{\Omega} \sum_{l=1}^{\mathrm{N}_{\mathrm{n}}}\left[\frac{1}{\left(d\left(\mu_{\mathrm{a}, l}(r)+\mu_{\mathrm{s}, l}(r)\right)\right)^{2}} \psi_{l}(r) \nabla \psi_{m}(r) \cdot \nabla \psi_{k}(r)\right] \mathrm{d} r+\int_{\Omega} \sum_{l=1}^{\mathrm{N}_{\mathrm{n}}} \psi_{l}(r) \psi_{m}(r) \nabla \psi_{k}(r) \mathrm{d} r \\
\frac{\partial \mathrm{K}}{\partial \mu_{\mathrm{s}}^{\prime}} & =\int_{\Omega} \sum_{l=1}^{\mathrm{N}_{\mathrm{n}}}\left[\frac{1}{\left(d\left(\mu_{\mathrm{a}, l}(r)+\mu_{\mathrm{s}, l}(r)\right)\right)^{2}} \psi_{l}(r) \nabla \psi_{m}(r) \cdot \nabla \psi_{k}(r)\right] \mathrm{d} r .
\end{aligned}
$$

Differentiating the FE-approximation for the truncated Fourier series approximation model, Eq. (18) with $\mu$, we obtain

$$
\frac{\partial \Phi^{h}(\omega)}{\partial \mu}=-\left[\mathrm{K}+\frac{i \omega}{\mathrm{c}} \mathrm{M}\right]^{-1} \frac{\partial \mathrm{K}}{\partial \mu} \Phi^{h}(\omega) .
$$

where $\frac{\partial \mathrm{K}}{\partial \mu}$ is as in Eqs. (A2),(A3).

\section{Appendix B: Reconstructions in a 3D mesh}

We generated time-resolved measurement data and carried out truncated Fourier series reconstructions in 3D. A mesh with 5413 nodes and 24064 elements, shown in Fig. B1 was used both simulating the data and solving the inverse problem in order to avoid modeling errors due to discretization. It should however be noted that this can lead to over optimistic results, and in practice, finer discretizations are required for a 3D problem especially close to the light sources. The computation times for the truncated Fourier series estimates using this mesh, with $\mathrm{N}_{\omega}=1, . .5$ frequencies were $418 \mathrm{~s}, 679 \mathrm{~s}, 770 \mathrm{~s}, 1436 \mathrm{~s}$ and $1607 \mathrm{~s}$ respectively.

\section{Appendix C: Comparison to Fourier transform model in Ref. [4]}

The model proposed in Ref. [4] can be stated as follows: the Fourier transform of the difference of two TD-DOT measurements, at a particular frequency, is equal to the difference in frequency domain measurements at that particular frequency. We compare here our proposed Fourier series approximation model (17), to the model proposed in Ref. [4], applied in absolute (not difference) imaging.

Let us consider a modified measurement vector $y_{\mathrm{Mod}}$, using the logarithm of the Fourier transformed measurement data $\Gamma(t)$, and assume negligible measurement noise $e \approx 0$. Following from Eq. (16), the modified measurement vector $y_{\mathrm{Mod}}$ is given by

$$
y_{\text {Mod }}=\left(\begin{array}{l}
\operatorname{Re} \log \mathcal{L}(\Gamma(t)) \\
\operatorname{Im} \log \mathcal{L}(\Gamma(t))
\end{array}\right)=\left(\begin{array}{l}
\operatorname{Re} \log \left(\Gamma\left(\omega_{k}\right)\right) \\
\operatorname{Im} \log \left(\Gamma\left(\omega_{k}\right)\right)
\end{array}\right)+\underbrace{\left(\begin{array}{l}
\operatorname{Re} \log (\mathcal{L}(Q(t))) \\
\operatorname{Im} \log (\mathcal{L}(Q(t)))
\end{array}\right)}_{y_{\text {Mod,s }}},
$$



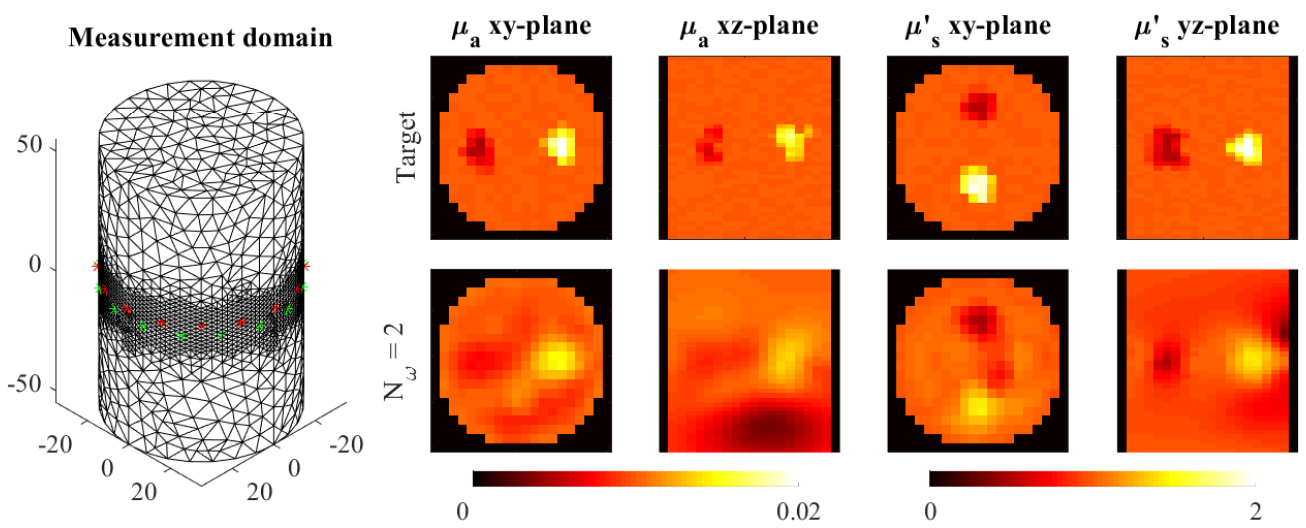

Fig. B1. Left image: The three-dimensional simulation domain with 16 sources (red stars) and 16 detectors (green stars) located at equi-spaced angular intervals on the waist of the cylinder. The dimensions shown are in millimeters. Right images: Target absorption and scattering distributions (top row) along xy-plane (at $\mathrm{z}=0$ ), $\mathrm{xz}$-plane (at $\mathrm{y}=0$ ) and yz-plane (at $\mathrm{x}=0$ ), and reconstructed distributions using two Fourier coefficients (bottom row) along the same planes.

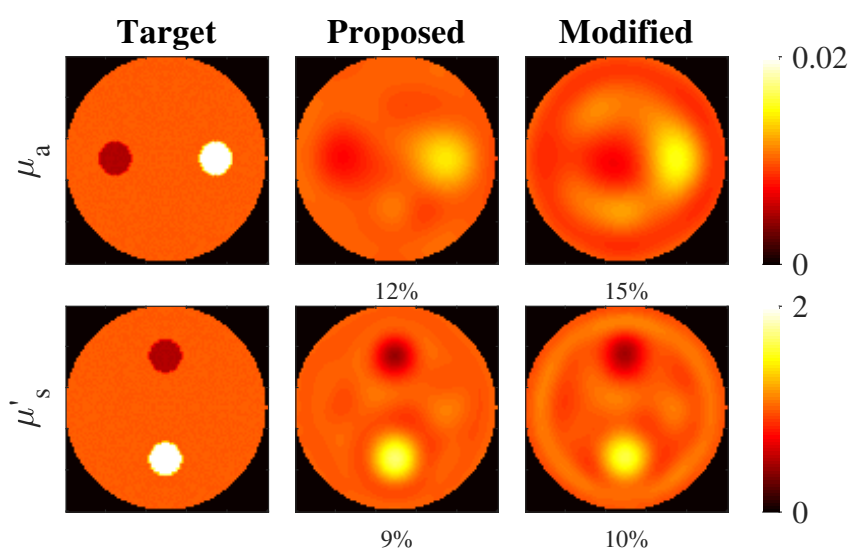

Fig. C1. Absorption (first row) and scattering (second row) distributions. First column: simulated (true) target. Second column: Estimates computed with the proposed model (17). Third column: estimates computed using using the modified model where $y_{\mathrm{Mod}, \mathrm{s}}$ was set as zero. One Fourier frequency $\left(\mathrm{N}_{\omega}=1\right)$ was used in both estimates.

where $y_{\text {Mod,s }}$ is the modified source Fourier transform. Considering difference imaging, where the measurements $y_{\text {Mod }}$ due to two different optical parameter distributions are subtracted, the source Fourier transform $y_{\text {Mod,s }}$ cancels out. As such, difference imaging with measurements $y_{\text {Mod }}$ is unaffected by the source characteristics, as carried out in Ref. [4]. Nevertheless for absolute imaging, the source Fourier transform $y_{\text {Mod,s }}$, needs to be included in the model. That is demonstrated in Fig. C1, where we the reconstruction accuracy of proposed measurement model (17), is compared to a modified measurement model where $y_{\text {Mod,s }}$ was set as zero.

We observe that ignoring the source Fourier transform, produces artefacts in the reconstructed parameters, due to incorrect modelling of the 10ps source pulse used in these simulation. TD-DOT instruments mentioned earlier [2,3,11,17], used pulse widths of 4-100ps. Hence, achieving absolute imaging with these instruments and ignoring the finite size pulse width, could cause 
similar artefacts in the estimated images. The results in Sec. 4 also indicate that using a few frequencies $(\sim 4)$ produces better estimates than using one frequency.

\section{Acknowledgments}

This work was supported by the Academy of Finland (projects 314411 and 312342 Centre of Excellence in Inverse Modelling and Imaging) and Jane and Aatos Erkko Foundation.

\section{References}

1. J. M. Cochran, D. R. Busch, L. Lin, D. L. Minkoff, M. Schweiger, S. Arridge, and A. G. Yodh, "Hybrid time-domain and continuous-wave diffuse optical tomography instrument with concurrent, clinical magnetic resonance imaging for breast cancer imaging," J. Biomed. Opt. 24, 051409 (2019).

2. J. C. Hebden, A. Gibson, R. M. Yusof, N. Everdell, E. M. Hillman, D. T. Delpy, S. R. Arridge, T. Austin, J. H. Meek, and J. S. Wyatt, "Three-dimensional optical tomography of the premature infant brain," Phys. Medicine \& Biol. 47, 4155 (2002).

3. T. Austin, A. Gibson, G. Branco, R. M. Yusof, S. Arridge, J. Meek, J. Wyatt, D. Delpy, and J. Hebden, “Three dimensional optical imaging of blood volume and oxygenation in the neonatal brain," Neuroimage 31, 1426-1433 (2006).

4. A. Gibson, T. Austin, N. Everdell, M. Schweiger, S. Arridge, J. Meek, J. Wyatt, D. Delpy, and J. Hebden, “Threedimensional whole-head optical tomography of passive motor evoked responses in the neonate," Neuroimage 30, 521-528 (2006)

5. J. Chen, "Optical tomography in small animals with time-resolved monte carlo methods," Diss. Abstr. Int. 74 (2012).

6. S. R. Arridge and W. R. B. Lionheart, "Nonuniqueness in diffusion-based optical tomography," Opt. Lett. 23, 882-884 (1998).

7. A. Gibson, J. Hebden, and S. R. Arridge, "Recent advances in diffuse optical imaging," Phys. Medicine \& Biol. 50, R1 (2005).

8. A. Pifferi, D. Contini, A. Dalla Mora, A. Farina, L. Spinelli, and A. Torricelli, "New frontiers in time-domain diffuse optics, a review," J. Biomed. Opt. 21, 091310 (2016).

9. S. R. Arridge and M. Schweiger, "Direct calculation of the moments of the distribution of photon time of flight in tissue with a finite-element method," Appl. Opt. 34, 2683-2687 (1995).

10. F. E. Schmidt, M. E. Fry, E. M. Hillman, J. C. Hebden, and D. T. Delpy, "A 32-channel time-resolved instrument for medical optical tomography,” Rev. Sci. Instruments 71, 256-265 (2000).

11. E. M. Hillman, J. C. Hebden, M. Schweiger, H. Dehghani, F. E. Schmidt, D. T. Delpy, and S. R. Arridge, “Time resolved optical tomography of the human forearm," Phys. Medicine \& Biol. 46, 1117 (2001).

12. L. C. Enfield, A. P. Gibson, N. L. Everdell, D. T. Delpy, M. Schweiger, S. R. Arridge, C. Richardson, M. Keshtgar, M. Douek, and J. C. Hebden, "Three-dimensional time-resolved optical mammography of the uncompressed breast," Appl. Opt. 46, 3628-3638 (2007)

13. L. C. Enfield, A. P. Gibson, J. C. Hebden, and M. Douek, "Optical tomography of breast cancer-monitoring response to primary medical therapy," Target. Oncol. 4, 219-233 (2009).

14. L. Enfield, G. Cantanhede, M. Douek, V. Ramalingam, A. Purushotham, J. C. Hebden, and A. P. Gibson, "Monitoring the response to neoadjuvant hormone therapy for locally advanced breast cancer using three-dimensional time-resolved optical mammography," J. Biomed. Opt. 18, 056012 (2013).

15. H. Eda, I. Oda, Y. Ito, Y. Wada, Y. Oikawa, Y. Tsunazawa, M. Takada, Y. Tsuchiya, Y. Yamashita, M. Oda, A. Sassaroli, Y. Yamada, and M. Tamura, "Multichannel time-resolved optical tomographic imaging system," Rev. Sci. Instruments 70, 3595-3602 (1999).

16. A. Farina, M. Betcke, L. Di Sieno, A. Bassi, N. Ducros, A. Pifferi, G. Valentini, S. Arridge, and C. D’Andrea, "Multiple-view diffuse optical tomography system based on time-domain compressive measurements," Opt. Lett. 42, 2822-2825 (2017).

17. B. Montcel, R. Chabrier, and P. Poulet, "Detection of cortical activation with time-resolved diffuse optical methods," Appl. Opt. 44, 1942-1947 (2005).

18. J. J. Selb, D. K. Joseph, and D. A. Boas, “Time-gated optical system for depth-resolved functional brain imaging," J. Biomed. Opt. 11, 044008 (2006).

19. D. Milej, A. Gerega, M. Kacprzak, P. Sawosz, W. Weigl, R. Maniewski, and A. Liebert, "Time-resolved multichannel optical system for assessment of brain oxygenation and perfusion by monitoring of diffuse reflectance and fluorescence," Opto-Electronics Rev. 22, 55-67 (2014).

20. F. Gao, H. Zhao, and Y. Yamada, "Improvement of image quality in diffuse optical tomography by use of full time-resolved data," Appl. Opt. 41, 778-791 (2002).

21. M. A. Naser and M. Deen, "Time-domain diffuse optical tomography using recursive direct method of calculating jacobian at selected temporal points,” Biomed. Phys. \& Eng. Express 1, 045207 (2015).

22. S. Okawa, Y. Hoshi, and Y. Yamada, "Improvement of image quality of time-domain diffuse optical tomography with lp sparsity regularization,” Biomed. Opt. Express 2, 3334-3348 (2011).

23. S. R. Arridge, “Optical tomography in medical imaging,” Inverse Probl. 15, R41 (1999). 
24. M. Schweiger and S. R. Arridge, "Application of temporal filters to time resolved data in optical tomography," Phys. Medicine \& Biol. 44, 1699 (1999).

25. J. Selb, A. M. Dale, and D. A. Boas, "Linear 3D reconstruction of time-domain diffuse optical imaging differential data: improved depth localization and lateral resolution," Opt. Express 15, 16400-16412 (2007).

26. A. Ishimaru, Wave Propagation and Scattering in Random Media (Academic, Newyork, 1978).

27. S. Arridge, M. Schweiger, M. Hiraoka, and D. Delpy, "A finite element approach to modelling photon transport in tissue," Med. Phys. 20, 299-309 (1993).

28. S. R. Arridge, "Photon-measurement density functions. part I: Analytical forms," Appl. Opt. 34, 7395-7409 (1995).

29. S. R. Arridge and M. Schweiger, "Photon-measurement density functions. part 2: Finite-element-method calculations," Appl. Opt. 34, 8026-8037 (1995).

30. A. Pulkkinen and T. Tarvainen, "Truncated fourier-series approximation of the time-domain radiative transfer equation using finite elements," J. Opt. Soc. Am. A 30, 470-478 (2013).

31. M. Mozumder, T. Tarvainen, S. R. Arridge, J. Kaipio, and V. Kolehmainen, "Compensation of optode sensitivity and position errors in diffuse optical tomography using the approximation error approach," Biomed. Opt. Express 4 , 2015-2031 (2013).

32. M. Mozumder, T. Tarvainen, J. P. Kaipio, S. R. Arridge, and V. Kolehmainen, "Compensation of modeling errors due to unknown domain boundary in diffuse optical tomography,” J. Opt. Soc. Am. A 31, 1847-1855 (2014)

33. J. Kaipio and E. Somersalo, Statistical and Computational Inverse Problems (Springer, Newyork, 2005).

34. C. E. Rasmussen, "Gaussian processes in machine learning," in Advanced lectures on machine learning, (Springer, 2004), pp. 63-71.

35. M. Schweiger and S. R. Arridge, "The Toast++ software suite for forward and inverse modeling in optical tomography," J. Biomed. Opt. 19, 040801 (2014). 